\title{
Rellenando espacios: hacia una ordenación de los sistemas de servicios sociales desde la respuesta a la complejidad
}

\author{
Joan Uribe Vilarrodona \\ Universidad de Barcelona y Consorci de Serveis Socials de Barcelona \\ joan.uribe@ub.edu
}

Gizarte-zerbitzuetako sistemen etorkizuneko erronka estrategikoen artean bi nagusitzen dira: bete beharreko lagun egitearen intentsitatea eta egoeren konplexutasunaren arta. Gainera, bi kontzeptuei buruzko kontsentsu eza dute oinarri, zeinak lagun egiteari buruzko irizpide-, adierazleeta eredu-kontsentsuaren gabezia dakar. Bestetik, ezin dira beste sistemekin erabili (osasuna, hezkuntza, enplegua). Barne-eremuari dagokionez, lehen- -edo oinarrizkoa-eta bigarren-mailako -edo espezializatua-arretaren arteko banaketa ezabatu beharko litzateke, pertsonaren interakziogaitasuna berreskuratzeko lagun egitearen mesedetan: eskubide subjektiboen ikuspegi batetik, pertsonaren eta haren premien eta prozesuaren intentsitate-premia eta konplexutasun-maila aintzat hartuko dituena; ez sistemak berezko duen logikari jarraituta.

\section{GAKO-HITZAK:}

Gizarte-bazterketa, gizarte-zerbitzuetako sistemak, lehen-mailako arreta, arreta espezializatua, intentsitatea, konplexutasuna, elkarreragingarritasuna.
Los sistemas de servicios sociales tienen en la intensidad del acompañamiento a realizar, así como en la atención a la complejidad de las situaciones, sus retos estratégicos de futuro. A su vez, estos parten de una falta de consenso en los conceptos clave, lo cual conlleva ausencia de consenso en los criterios, indicadores y modelos de acompañamiento. Tampoco son interoperables con otros sistemas (sanitario, educativo, empleo). A nivel interno, la subdivisión entre atención primaria -0 básica- y secundaria - o especializada-, debería ser superada en aras de un acompañamiento basado en la recuperación de la capacidad de interacción de la persona, desde una perspectiva de derechos subjetivos, que contemple la necesidad de cada intensidad y nivel de complejidad desde la persona, su necesidad y proceso, y no desde la lógica del sistema en sí mismo.

\section{Palabras clave:}

Exclusión social, sistemas de servicios sociales, atención primaria, atención especializada, intensidad, complejidad, interoperatibilidad. 


\section{Introducción}

Los sistemas de servicios sociales tienen la misión de dar respuesta a determinadas situaciones que, aún, se fundamentan a partir de definiciones lábiles, de conceptos frecuentemente pendientes de consenso o carentes de demostración empírica. Esta circunstancia dificulta enormemente la homologación y coordinación entre sistemas sociales distintos, así como entre niveles de los mismos sistemas, y la interoperatibilidad con sistemas distintos de los sociales (salud, educación, empleo, entre otros). Es así con conceptos como el de exclusión social (en las distintas gradaciones de exclusión social -leve, moderada, grave-, así como del riesgo de exclusión social); y, en los últimos tiempos, y a tenor de la necesidad de nombrar y conceptualizar nuevas necesidades, el de complejidad. Esta realidad contrasta con la de otros sistemas, como por ejemplo los educativos y sanitarios, que suelen tener herramientas que operativizan su actuación a partir de definiciones y de delimitar el alcance de su actuación a partir de ellas, objetivando así las necesidades y relacionando estas con sus carteras de servicios y circuitos de actuación.

Cabe destacar que todo ello es posible porque su anclaje está precisamente en la parametrización de los estándares y necesidades educativas en el primer caso, y de la objetivación de la salud y estratificación y graduación de la ausencia de esta, en el segundo. Eso no es así para los sistemas de protección social, en tanto que la definición del estándar social acaba resultando inaprensible. La simultaneidad de realidades y situaciones sociales en relación con la subjetividad inherente a la definición de estándares en base a los cuales definir qué está sujeto a la necesidad de un sistema de protección social de apoyo, y su carácter cambiante, lo hace muy difícil. Si a ello le sumamos la incapacidad histórica que ha tenido la sociedad para generar sistemas de protección social con bases asentadas en el estudio y análisis sistemático de datos orientados a la comprensión de las realidades cambiantes y a su categorización y parametrización, tenemos como resultado el permanente desajuste entre las necesidades sociales que surgen a partir de las realidades cambiantes, y el diseño y respuesta de los sistemas de servicios sociales que, por otra parte, no han dejado aún de orientarse básicamente a la reacción ante la necesidad presente y no a la planificación futura.

Con la intención de mejorar la posición de los sistemas de servicios sociales en este sentido, se pretende presentar aquí el concepto de exclusión social a partir de su evolución histórica. También algunas herramientas para su medición, así como un sucinto análisis de los marcos conceptuales de dichas herramientas. A su vez, se ofrecerá una aproximación a los conceptos de interacción y de complejidad y su vinculación al de exclusión social. A partir de todo ello, este texto quiere proponer una adecuación de los sistemas de servicios sociales a las nuevas necesidades, considerando también el papel de la complejidad como eje vertebral y comunicador de estos sistemas, superando la actual concepción dual de niveles de servicios sociales básico/primaria y especializada/secundaria, e intentando facilitar la articulación de un sistema de protección social estratificado, coordinado y replicable, con el objeto de ser eficiente en el establecimiento de relaciones eficaces y eficientes entre todos los subsistemas y circuitos relacionados a todo sistema social - tanto a nivel interno de cada sistema, como entre niveles (básico, especializado), entre sistemas socialeso con sistemas distintos, como los sanitarios o educativos (interoperatibilidad).

Igualmente, incluye una aproximación que incorpore la flexibilidad en los circuitos e itinerarios de atención basados en las necesidades de las personas, y que mejore en lo posible la respuesta en relación a los actuales ámbitos dentro de los sistemas sociales (vulnerabilidad, desamparo, infancia, discapacidad, dependencia, exclusión social, riesgo de exclusión social, etc.), orientada a facilitar un diseño de sistemas de servicios sociales organizados en tanto que sistemas compatibles, coordinables e interoperables con otros sistemas.

\section{Exclusión social}

\subsection{El concepto y sus definiciones}

No hay consenso sobre qué es aquello que el concepto de exclusión social pretende explicar y que, además, se ubica en un contexto social cambiante. Por ello, proponemos aquí algunas aproximaciones que deben ser contextualizadas con relación al momento en que cada conceptualización apareció. A su vez, todas ellas, de una u otra forma, están aún vigentes en la actualidad. La primera es la de René Lenoir quien, en la década de los años 1970, definió a los excluidos - "les exclús"- como todos los desalojados al margen del crecimiento económico, visión que aumentaba sensiblemente los márgenes de la tradicional percepción de la pobreza y la inadaptación, ampliando el campo de reflexión vigente hasta el momento: su aportación fue entender que categorías como "desviados, anormales, inadaptados o marginados" era insuficiente, incluso incorrecta, al darse cuenta de que el nudo de la cuestión debía entenderse a través de la creciente dimensión de poblaciones "excluidas" de o en una sociedad cada vez más enriquecida, de confort. Dicho de otra forma, la propuesta de Lenoir propició comenzar a pensar la exclusión como proceso social y económicamente producido.

No obstante, su enfoque, desde un posicionamiento liberal, no fue más allá: entendía que en cualquier caso la situación se generaba a partir de la falta de medios personales (competenciales, materiales, sociales, culturales) de los "inadaptados", no abordaba las causas económicas de la pobreza ni de relación con el acceso a ingresos económicos 
suficientes vinculada a la exclusión y se centraba en proponer un abordaje que "insertase", a través, básicamente, de una acción social coordinada y eficaz, sentando las bases de un modelo centrado en la gestión del problema desde el sistema de protección social, obviando el abordaje de las causas, inherente a otros sistemas y marcos, distintos en gran medida del de protección social (García, 2013).

Siguiendo con la construcción del concepto, Robert Castel formuló una propuesta en la que "la exclusión" no sería en ningún caso una ausencia de relación social, sino un conjunto de relaciones sociales particulares con la sociedad como un todo (Castel, 1997). Dicho de otra manera, según Castel no hay nadie que esté fuera de la sociedad, sino un conjunto de posiciones cuyas relaciones con el centro son más o menos laxas: algunos ejemplos son las personas extrabajadoras víctimas del desempleo prolongado, jóvenes que no encuentran trabajo, poblaciones mal escolarizadas, mal consideradas, con mala vivienda y mala atención sanitaria. En su análisis de la obra de Castel, Martínez Veiga dice al respecto que hay tres elementos centrales en la propuesta de Castel: el primero y básico es la afirmación de que la exclusión tiene una dimensión relacional básica. El segundo, que la exclusión implica un "aflojamiento" de las relaciones con la comunidad local, con los servicios e instituciones estatales y con la sociedad a la que la persona pertenece, así como con la familia y amigos. En tercer lugar, subraya que el origen del fenómeno de la exclusión está en la sociedad salarial. Aun para los que están arrojados fuera el empleo, el origen de esta situación hay que buscarla en una reorganización de ella. Concluye que "la exclusión social se ve como fruto de dos fenómenos, la ausencia de trabajo y la ruptura de las relaciones sociales” (Martínez Veiga, 2008: 12).

Como resumen, podríamos decir que los ejes de articulación de la exclusión social pivotarían alrededor de, por un lado, el acceso a bienes materiales a través de los medios disponibles desde la sociedad salarial -o mejor dicho, la ausencia de acceso a ellos-, y, por otro, de la existencia y capacidad suficiente de respuesta del sistema relacional, ya sean estos sistemas relacionales la sociedad, la comunidad, el sistema de protección formal, la familia, los amigos, u otros. Estos dos ejes, a su vez, tienen en su zona intermedia el espacio de la vulnerabilidad que es también de difícil aprehensión, puesto que tiene límites cambiantes en base a cada circunstancia personal en relación a los dos ejes, así como a cada circunstancia de contexto, cambiante también: el gradiente de intensidad que definen uno y otro imposibilitan acotar de forma genérica los espacios de la vulnerabildad, pudiendo a lo sumo aproximarlos.

La tercera aproximación al concepto la tomamos de Brugué, Gomà y Subirats, quienes en 2002, definieron exclusión social como "concepto que engloba a la pobreza pero va más allá; la exclusión social se define también por la imposibilidad o dificultad intensa de acceder a los mecanismos de desarrollo personal e inserción sociocomunitaria y a los sistemas preestablecidos de protección" (Brugué et al., 2002: 9). Estos autores, integrando la doble perspectiva propuesta por Castel, toman en consideración de forma explícita el desarrollo de la propia persona sintonizando con enfoques como el de Amartya Sen, entre otros. El conjunto de la definición considera de forma implícita el acceso a los medios económicos y explicita la inserción sociocomunitaria, aproximando lo que Castel define como sistema relacional a una concepción abierta a través del paraguas de lo social y lo comunitario. Añade, como aportación de especial importancia, los sistemas de protección.

A su vez, entienden la exclusión social como fenómeno estructural, dinámico -en línea con la propuesta de su condición procesual, elaborada ya por Castel y ampliamente aceptada hoy en día-, y en tanto que fenómeno politizable, en el sentido de ser susceptible de generación de activación política en las personas que son consideradas como excluidas sociales. Consideran también que los mecanismos generadores de exclusión son la fragmentación tridimensional de la sociedad -en relación con la condición especialmente compleja y fragmentada hoy en día-, el impacto sobre el empleo de la economía postindustrial, y como tercer factor, el propio déficit de inclusividad del Estado del Bienestar (Brugué et al., 2002: 10). También interesa la conceptualización elaborada por parte de algunas administraciones públicas. Así, el Diccionari de Serveis Socials publicado por la Generalitat de Catalunya, define exclusión social como: "Situación resultante de la acción ejercida por una sociedad que rechaza una o más personas o colectivos por su situación de dependencia física, psíquica o económica, con la subsiguiente desigualdad en el acceso a los recursos necesarios para una buena calidad de vida" (Generalitat de Catalunya, 2010: 77). A su vez, en 2018, esta misma Administración Pública define exclusión social a través de la página web del Departament de Treball, Afers Socials i Famílies (DTASF), bajo el título "Pobresa i inclusió social" de la siguiente manera: "el resultado de un proceso dinámico de acumulación y combinación de factores diversos de desventaja, vinculados a diferentes aspectos de la vida personal, social, cultural y política de los individuos" (DTASF, sin fecha) ${ }^{1}$. La definición se complementa afirmando en el texto que la integración social de una persona implica su reconocimiento y aceptación en los ámbitos económico, laboral, social, comunitario, de ciudadanía y de participación.

El Ayuntamiento de Barcelona posiciona también el concepto en el número 13 de la revista Barcelona Societat y a través de diversos artículos del volumen,

${ }^{1}$ El entrecomillado aparece en el original. No se ha podido establecer la autoría de la cita que utiliza el DTASF para posicionar el concepto. 
de los cuales destaca el mapa de conocimiento sobre la exclusión social (Jaumandreu, 2005), un mapa conceptual que expone la génesis y evolución del concepto y que abunda en la definición y factores ya citados de Brugué, Gomà y Subirats, que también publican en el monográfico. Por otra parte, la Estrategia de Inclusión y de Reducción de las Desigualdades Sociales de Barcelona (20172027) elaborada por el Ayuntamiento de Barcelona, dedica más de quince páginas a presentar de manera exhaustiva y detallada las formas de desarrollo de la exclusión en la ciudad en los últimos años, identificando causas y analizando procesos de cambio, sin entrar en la definición en sí misma.

A partir de las definiciones presentadas, no resulta difícil entender la dificultad que implica "fijar" de alguna manera la exclusión social y construir umbrales y límites claros, así como establecer indicadores objetivos e irrevocables para identificar las situaciones en que se materializa. A su vez, esta misma labilidad fragiliza la posibilidad de construir indicadores alrededor de este concepto a partir de conceptos como inclusión, vulnerabilidad, o su gradación (riesgo de exclusión social). ¿Cómo, pues, estratificar y fijar el escalamiento de unas a otras situaciones para, a partir de ello, sistematizar no solo la respuesta concreta, sino también la coordinación flexible y eficiente entre unas y otras, y hacerlo en el interior de los propios sistemas de servicios sociales, así como entre estos y aquellos con los que debe coordinarse (salud, educación y otros)? Veamos algunas herramientas de medición de la exclusión social con la intención de que puedan aportar alguna luz en este sentido.

\subsection{Algunas herramientas para la detección y categorización de la exclusión social}

Como apunta Fantova "fortalecer la capacidad de diagnóstico, valoración y evaluación resulta crítico y depende en buena medida de la capacidad de las disciplinas científicas y técnicas involucradas en la intervención social de aportar o desarrollar herramientas e instrumentos capaces de identificar y medir necesidades y situaciones a partir de conceptos y constructos rigurosos y contrastados, bien enfocados y delimitados. Herramientas e instrumentos fiables y válidos, que aporten cada vez mayor valor añadido en el proceso que comienza con la recogida de información y desemboca en el juicio profesional que antecede a la prescripción facultativa" (Fantova, 2017: 14). Hay algunas herramientas con propuestas interesantes que han avanzado, nos atreveríamos a decir que heroicamente, en el intento. Manzano cita algunas (Manzano, 2018), de las que destacamos: la matriz de autosuficiencia SSM (Self Sufficiency Matrix) ${ }^{2}$, una herramienta holandesa que, según este autor, estaría siendo adaptada en Catalunya; la Herramienta

${ }^{2}$ Self Sufficiency Matrix (SSM): http://www.selfsufficiencymatrix.org/zrm-int.aspx de Valoración Social para los Servicios Sociales Básicos en Cataluña DS-DIBA de la Diputación de Barcelona (DIBA) 3 ; y el Instrumento de Valoración de la Exclusión Social del Gobierno Vasco, aprobado por decreto en 2013. Comentaremos también alguna más, como Outcome Star o el sistema de indicadores de FOESSA, para identificar a las personas excluidas y cuantificar las dimensiones de los procesos de exclusión (Laparra y Pérez, 2008: 43-62).

\subsubsection{Sistema de indicadores para identificar a las personas excluidas y cuantificar las dimensiones de los procesos de exclusión (FOESSA)}

La fundación FOESSA, a través de sus informes sobre exclusión social, utiliza un sistema de indicadores que, si bien tiene un carácter y orientación básicamente sociológico y no orientado a la intervención social, ocupa un papel referente: los indicadores dan respuesta a los ejes hoy consensuados sobre los que pivota la exclusión social-económico, político y social relacional-. A su vez, a través del estudio longitudinal de datos que han ido ofreciendo y ofrecen, de carácter estatal, autonómico, o supramunicipal, permiten evidenciar y objetivar la existencia, evolución e incidencia en la sociedad de la exclusión social.

Este sistema de indicadores trabaja dimensiones y aspectos a través de tres ejes (económico, político y social/relacional). Las dimensiones del eje económico son la participación en la producción y en el consumo, y sus aspectos, la exclusión de la relacional salarial normalizada, la pobreza económica y la privación. Las dimensiones del eje político son las ciudadanías política y social, y los aspectos, el acceso efectivo a los derechos políticos, el abstencionismo y pasividad política, y el acceso limitado a los sistemas de protección social: sanidad, vivienda y educación. Por su parte, las dimensiones del eje social/relacional son la ausencia de lazos sociales y las relaciones sociales "perversas", siendo sus aspectos la integración en redes sociales “desviadas", la conflictividad social - conductas anómicas-y conflictividad familiar - violencia doméstica-(García et al., 2008: 46).

\subsubsection{Self Sufficiency Matrix (SSM)}

El manual para la aplicación de la Self Sufficiency Matrix define la self sufficiency (autosuficiencia) afirmando que: "Usted puede ser considerado autosuficiente cuando ha alcanzado un nivel aceptable de funcionalidad en los ámbitos en los que usted y los demás ciudadanos holandeses, confluyen en la vida diaria. [...] La autosuficiencia no es tan solo suficiencia por uno mismo. Para alcanzar o retener un nivel aceptable de funcionamiento, usted puede o debe hacer uso de las habilidades, la experiencia y las posibilidades de los demás. Estas otras personas, 
pueden ser familiares o amigos, pero también trabajadores de atención profesional que pueden ayudar en el objetivo de alcanzar niveles aceptables de funcionamiento" (Self Sufficiency Matrix, 2013: 5) . $^{4}$

Esta definición guarda elementos en común con el concepto de interacción, propuesto por Fantova y que este autor basa a partir de "considerar la interacción (humana) como el bien protegible, la finalidad específica, la necesidad de referencia para los servicios sociales" (Fantova, 2008: 42). Define el concepto como "el ajuste dinámico entre la capacidad de desenvolvimiento autónomo de la persona en sus entornos vitales y el apoyo social disponible a través de los vínculos familiares, convivenciales, comunitarios o relacionales en general. Proponemos la palabra interacción porque permite hacer referencia tanto a lo que la persona hace como al apoyo o ayuda que recibe gracias a los vínculos naturales que mantiene", y en tanto que propuesta que trascienda, adaptándolo al momento actual, al concepto de exclusión social.

Volviendo a la herramienta SSM y su objetivo, se nos explica en el citado manual que no es otro que estipular el nivel de autosuficiencia de la persona. Para hacerlo, establece un total de once áreas de exploración: capacidad económica, actividades diarias, vivienda, relaciones del ámbito doméstico, salud mental, salud física, adicciones, actividades de la vida diaria, redes sociales, participación comunitaria y relación con causas judiciales. Cada una de ellas centrada a través de los conceptos vinculados a cada ámbito y desarrolladas en un formato de puntuación de cada uno de ellos. La recogida de información se realiza a partir de tres fuentes: la primera y principal proveedora, la propia persona objeto de la evaluación a través de entrevista. La segunda, el personal profesional vinculado a la persona. La tercera, los sistemas de información disponibles y con información relevante, al alcance de la profesional evaluadora.

Resulta revelador el hecho de que sea una herramienta del sistema de salud pública. Más allá de las particularidades de Holanda con relación a su ordenación de sistemas en la conexión entre la respuesta social y sanitaria, resulta interesante el aterrizaje que esta herramienta lleva a cabo de la vigente definición de salud por parte de la Organización Mundial de la Salud (OMS), que define esta como el estado de completo bienestar físico, mental y social y no sólo la ausencia de afecciones o enfermedades. Es a partir de ella que la SSM integra como un todo - encarnado por cada persona como unidad indivisible - ocho ámbitos del dominio puramente social: capacidad económica, actividades diarias, vivienda, relaciones del ámbito doméstico, actividades de la vida diaria, redes sociales, participación comunitaria y relación con causas judiciales; y los tres restantes, salud mental,

${ }^{4}$ Esta y siguientes citas relativas a la herramienta SSM han sido traducidas del inglés por el autor. salud física y adicciones, de salud aunque con una clara vinculación con lo social. Esta herramienta da a entender que el éxito en el acompañamiento de las personas pasa por trabajar de manera integrada ambos aspectos, social y sanitario.

\subsubsection{Outcome Star}

Es interesante observar hasta qué punto el SSM tiene elementos en común con otra herramienta, Outcome Star, una de las versiones de la cual es la medición de evolución de la situación de personas en situación de sinhogar. Una herramienta creada y comercializada en el Reino Unido y que ha ido generando adhesiones en el ámbito del acompañamiento social. Puede que uno de los motivos de los puntos en común entre una y otra herramienta -es decir, de la integración de lo estrictamente social y lo estrictamente sanitario, concretando sobre cada individuo una atención centrada en la persona- esté en que la alta especificidad que requiere acompañar a personas en esta situación, combinada con la exagerada falta de recursos y circuitos de interoperatibilidad suele implicar modelos de acompañamiento como el Assertive Community Treatment, o Tratamiento Asertivo Comunitario (ACT por sus siglas en inglés) -equipos integrados de ámbito social y sanitario-, o el Intensive Case Management o Gestión de Casos (ICM por sus siglas en inglés) - gestor referente, normalmente del ámbito social, que coordina con los otros ámbitos con un alto nivel de eficiencia esperado en la respuesta- (Llobet et al., 2016: 23-24): modelos distintos pero, en cualquier caso, originados por la evidencia de la necesidad del abordaje integrado.

Los diez ámbitos de exploración de Outcome Star son: motivación y responsabilidad; autocuidado y habilidades para la vida; administración económica y administración personal; redes sociales y relaciones; uso indebido de drogas y alcohol; salud física; salud mental y emocional; gestión del tiempo; tenencia y administración de vivienda; e infracciones. La herramienta se valora a partir de un listado que define para cada ámbito y de forma detallada cada una de las once situaciones posibles a puntuar de o a 10. Se realizan dos mediciones simultáneas por persona, una realizada por la persona en situación de sin hogar - autoevaluación-y otra por una profesional, y se deben compartir ambas para así apreciar las diferencias de percepción. Se repite en el tiempo, con lo que se puede dar seguimiento a la evolución del proceso de la persona.

Como resumen, destacaríamos de la herramienta SSM la hibridación de lo social y sanitario en una exploración y evaluación orientada a un acompañamiento integrado y coordinado entre los dos sistemas. A su vez, el SSM, de la misma manera que Outcome Star, ejemplifica la búsqueda de herramientas conjuntas orientadas al común objetivo centrado en la persona y evidencia la necesidad de compartir, consensuar y categorizar 
ambos sistemas - sanitario y social- de forma si no idéntica, al menos equiparable en la sistematización de conceptos: su definición, límites, indicadores de evaluación y de resultado, así como resultados esperados.

\subsubsection{Herramienta de valoración social para los servicios sociales básicos en Cataluña DS-DIBA}

Otra de las herramientas es la Herramienta de Valoración Social para los Servicios Sociales Básicos en Cataluña DS-DIBA ${ }^{5}$, orientada a identificar y estratificar grupos de riesgo entre las personas usuarias en base a la gravedad de sus situaciones sociales. Su objetivo es valorar las necesidades sociales, discriminar situaciones a atender, así como su prioridad e intensidad a través de una atención personalizada y buscando una equidad en el contexto del sistema catalán de servicios sociales básicos.

La herramienta identifica treinta y tres situaciones sociales que deben evidenciarse - objetivarse-, en relación con cuarenta y un factores indiciarios del riesgo a la protección, repartidas en tres categorías: autonomía funcional, autonomía material o instrumental, autonomía relacional. Con el fin de determinar las necesidades, la herramienta orienta a una estratificación escalonada a través de las categorías: vulnerable, riesgo, alto riesgo. No disponemos de la información suficiente sobre esta herramienta como para avanzar conclusiones que sin duda serían aventuradas. No obstante, la información proporcionada por el póster de la Diputación de Barcelona habla de una herramienta desarrollada en el transcurso de una investigación-acción de cuatro años con la participación de doscientos cincuenta expertos y nueve ayuntamientos y a partir de la valoración de casos, lo cual orienta a una conexión probable de la herramienta con las necesidades tanto sociales como de ajuste de estas a los recursos y sistemas de los sistemas sociales de los municipios.

Por otra parte, parece ser una herramienta orientada exclusivamente a los servicios sociales básicos, con el objetivo de dar respuestas a las necesidades de las administraciones que la fomentan municipios-. Puede que por ello adolezca de una de las habituales carencias de los sistemas de protección social: una visión transversal más allá de las lógicas de organización y actuación básico/ especializado para así poder centrar el sistema en la persona y las posibles complejidades que fluctúan y se manejan con dificultad entre lo que hoy son los dos compartimentos estancos, el básico y el especializado.

\subsubsection{Instrumento de Valoración de la Exclusión Social (IVES) del Gobierno Vasco}

Vamos ahora al Instrumento de Valoración de la Exclusión Social del Gobierno Vasco, analizado a través del Decreto 385/2013, de 16 de julio, de aprobación del instrumento de valoración del País Vasco. Es importante situar esta herramienta en el contexto del trabajo de revisión realizado por el sistema de protección social vasco que en 2015 se orientó a una reordenación de la red de recursos y programas para la inserción social en Gipuzkoa, a través del cual se llevaron a cabo propuestas genéricas de mejora (SIIS, 2015). El objetivo de esta revisión era orientar un cambio de marco conceptual centrado en dos aspectos: superar diseños enfocados al supuesto merecimiento de la persona al apoyo, para centrar la acción a partir del derecho subjetivo, y reformular ámbitos competenciales para dar una mejor respuesta a la necesidad de acompañamiento social a partir de su intensidad.

Esta herramienta se orienta tanto a los servicios sociales de primaria como a los de secundaria - lo cual apunta a un diseño de sistemas abierto a la coordinación entre uno y otro nivel del sistema-. Resulta de especial interés lo que parece ser la base conceptual que otorga carta de naturaleza a esta conexión entre primaria y secundaria: el decreto define la inclusión social y la prevención del riesgo de exclusión social como el objeto de los servicios sociales de atención primaria. Y para los de secundaria, las necesidades derivadas de las situaciones de exclusión social. Se formaliza como una propuesta de unificación conceptual tendente a facilitar una estratificación y coordinación entre ámbitos y sistemas, como refleja el decreto en su presentación cuando afirma que el instrumento: "servirá a los servicios sociales municipales y forales para valorar con criterios comunes la existencia de situaciones de exclusión social -en diferentes niveles de intensidad leve, moderada y grave- o de riesgo de exclusión social”.

Comparando esta propuesta con la de la ley catalana de servicios sociales, Ley 12/2007 de 11 de octubre, constatamos que Cataluña distribuye la respuesta del sistema de protección social entre servicios sociales básicos y servicios sociales especializados, en paralelismo con la organización del sistema vasco. En cambio, mientras el País Vasco apuesta por una respuesta que parte de una integración basada en la exclusión social, Cataluña, que centra una única misión para el conjunto de sistemas de servicios sociales en su artículo 3.2, al definir las necesidades sociales como las que "repercuten en la autonomía personal y el soporte a la dependencia, en una mejor calidad de vida personal, familiar y grupal, en las relaciones interpersonales y sociales y en el bienestar de la colectividad", aterriza el detalle de los objetivos de los niveles básico y especializado con una orientación muy diferenciada entre uno y otro, estableciendo en todo caso y cuando sea necesario coordinaciones en las situaciones puente de tránsito 
entre básico y especializado, pero partiendo de una dedicación altamente diferenciada entre los dos.

Se entiende pues que el sistema público vasco apuesta por agrupar bajo el paraguas de la exclusión social todas las situaciones tributarias de la acción de su sistema de protección social, vinculando a este paraguas conceptual -la exclusión social-, otras como dependencia, desamparo, discapacidad, por citar algunas de las más habituales, con una genérica y a partir de la que articular el sistema de protección social, y que sería la inclusión/exclusión social. Es por ello un enfoque interesante a ser estudiado a partir de la valoración que realicen tanto las personas beneficiarias como las profesionales, así como el propio sistema vasco de protección social.

Volviendo al Instrumento de Valoración de la Exclusión Social del Gobierno Vasco, las categorías diagnósticas que define son: la inclusión social -entendida como "[...] el acceso adecuado a los sistemas de protección social existentes, y con la facilidad que tienen las personas para utilizar la información precisa para acceder a los derechos y deberes que como ciudadanas les corresponden. Tiene que ver con sus capacidades personales y sociales para relacionarse y con la participación social"-; el riesgo de exclusión social -como "la presencia o ausencia de una serie de factores 0 características personales asociadas que hacen que aumenten las probabilidades de aparición de consecuencias adversas que deriven en situaciones o procesos de exclusión social"-; exclusión social, "cuando sus condiciones de vida y convivencia se están viendo afectadas por múltiples carencias que persisten en el tiempo. Al acumularse provocan la existencia de una situación de exclusión social que está relacionada directamente con los recursos personales, los recursos relacionales y los recursos materiales" (BOPV, 2013).

La intensidad de la exclusión social ordena esta en leve, moderada y grave. La exclusión social leve implica "desarraigo inicial o leve. Personas que disponen de contactos frágiles con sus familiares o red de apoyo". La exclusión social moderada supone una "fase avanzada de desarraigo. Personas que han roto sus lazos sociales básicos”. La exclusión social grave, comporta que la persona "no participa en la sociedad. Precariedad o ausencia de relaciones sociales y afectivas" (BOPV, 2013). Los indicadores de la herramienta se organizan en cinco categorías: económica, laboral y residencial; convivencial; personal; salud; y social. Cada indicador es puntuado con valores entre 0,1 y 2 , siendo o una "situación positiva o, cuando menos, no problemática: en zona de inclusión"; 1: "precariedad o ciertas dificultades. Zona de exclusión”; 2: "privación y problemas graves. Zona de exclusión” (BOPV, 2013). La valoración se realiza a partir de dos criterios: “Criterio 1: se refiere a las carencias personales y relacionales. Valoramos si carece de apoyo social proveniente de redes primarias y secundarias, así como de recursos personales. Se tienen en cuenta indicadores de los ámbitos convivencial, personal y social. Criterio 2: relativo a la "merma de la autonomía personal y económica, valora indicadores de los ámbitos económico-laboral, convivencial, personal y de la salud", criterios que se ponderan al detalle y se aplican a través de un desarrollo detallado de cada uno de los cinco indicadores, tal y como se define en el propio decreto.

Parece que esta herramienta cuenta en su haber con éxitos, así como con aspectos de mejora: los servicios forales la han incorporado como herramienta básica, mientras que los municipales no terminan de integrarla como tal (Orbegozo, 2016). En cualquier caso, al objeto de este artículo, este instrumento de valoración materializa la disposición de una única herramienta común a los dos niveles del sistema (llamémoslo municipal-foral o básico-especializado), a partir de lo que se intuye como un consenso en el encaje de los dos niveles del sistema de protección social, y que pivotaría siempre con relación a situaciones vinculadas a la exclusión social. Así, las dos partes del sistema no se diferenciarían en relación con su vinculación a una cartera distinta de responsabilidades, sino que se caracterizarían por la capacidad de respuesta a la complejidad e intensidad del acompañamiento social: inclusión y prevención -la básica-; respuesta a las necesidades de la exclusión -la foral-, con independencia en ambos casos de si se trata de niños y niñas en desamparo, personas mayores en grado de dependencia, o personas víctimas de violencia machista, por poner algunos ejemplos.

Un análisis reciente a este trabajo realizado desde Gipuzkoa (Larrión et al., 2018), reconoce la dificultad de llevar a cabo una reordenación de un sistema ya en funcionamiento, así como la importancia de una cartera de servicios y centros alineados a ella, y con una definición homogénea. También la dificultad de asimilar el abordaje a las situaciones de exclusión a las situaciones en las que se atiende dependencia o desprotección: serían, aún, espacios con poca interconexión y con poca permeabilidad. A su vez, en el marco de la intervención en el marco de la exclusión social, se destaca como dificultad importante la inexistencia de un modelo común de intervención (Larrión et al., 2018: 63). También, destacan que el trabajo realizado a partir de la revisión iniciada unos años antes ha posibilitado disponer de un catálogo de servicios más ordenado y más orientado al derecho subjetivo, sentando las bases para la creación de una red de servicios sociales más orientada a la autonomía, derechos y calidad de vida de las personas (Larrión et al., 2018: 64), uno de los objetivos fundamentales que debe cubrir todo sistema de servicios sociales.

A partir de estos avances, el debate estaría ahora en cómo conseguir acabar de difuminar o neutralizar la separación de acompañamiento en base a su intensidad, e ir abordando el deslinde de fronteras entre subsistemas en función de si existen o no, 
vinculadas a la exclusión social, situaciones de desprotección y/o dependencia en un sentido amplio. Así pues, parece que este enfoque hacia el que se orienta el País Vasco puede tener mucho que aportar a la actual necesidad de articulación de los sistemas de servicios sociales desde criterios de complejidad y a partir de modelos de atención centrados en la persona.

\subsection{Algunas conclusiones relativas al concepto de exclusión social}

El recorrido histórico del concepto refleja la evolución y cambio de las situaciones que describe. Hoy se puede dar por consensuada la visión integrada de tres vectores causales de las situaciones de exclusión social: la imposibilidad de acceso a la sociedad salarial de la que nos habla Castel, entendida como la capacidad de desenvolverse en criterios de equidad a nivel económico; la ausencia de tejido y estructura social suficiente para el mantenimiento de la autonomía y capacidades deseadas; y la insuficiente capacidad del estado del bienestar para garantizar sociedades que cumplan con los criterios de justicia social en base al cumplimiento de los derechos sociales.

También hoy parece indiscutible destacar la complejidad como un elemento crítico de la exclusión social, puesto que incide en la capacidad de aprehender y, por tanto, comprender, detectary responder a estas situaciones. Esta complejidad se expresa en distintos niveles. Como apuntan Brugué, Gomà y Subirats, la fragmentación de la sociedad implica una creciente división de fórmulas, sistemas y necesidades sociales que aísla a individuos y grupos reduce la capacidad de reacción ante los problemas al no disponer de alianzas suficientes, y contribuye al desconocimiento de las situaciones sociales ajenas a las propias; la economía postindustrial, entendida como un "modelo de economía informacional con esquemas de producción postfordistas” (Brugué et al., 2002: 10), reduce al máximo la capacidad para alcanzar niveles de autonomía y libertad a nivel individual en cada vez más segmentos de la sociedad; un marco de creciente presión neoliberal a la organización de los Estados, que se orientan cada vez más hacia el recorte o la escatimación del reconocimiento de determinados derechos, a veces aduciendo la necesidad de hacerlo para cumplir con otros derechos, cuando no recurriendo a la criminalización de la exclusión; la creciente debilidad y pérdida de capacidades alcanzadas en términos de estado del bienestar, que aumenta vertiginosamente la fragilidad de porciones cada vez más numerosas de población que disponen de menos apoyo de los Estados para contener y solventar las situaciones de vulnerabilidad que les afectan.

A esos factores, que individualmente o combinados generan de por sí muchas y distintas circunstancias de exclusión social que convierten en más y más complejo su abordaje, hay que sumar otros, de reciente aparición y consolidación como el incremento de la expectativa de vida de la población, combinada con el deterioro de la salud, y relacionado con la capacidad de mantenimiento económico de la situación de cada persona. Por otra parte, parece claro que no está claro qué queremos abordar cuando hablamos de exclusión social. 0, en cualquier caso, parece claro que no está consensuado. Los sistemas de servicios sociales están organizados, por mandato legal, en forma de sistemas que responden reactivamente a situaciones de pobreza, de excepcionalidad social sin recursos de autogestión, o ambas en coincidencia, desde un abordaje paliativo. Como apunta Aguilar, sometidos a la demanda y a su reacción, y por tanto sin capacidad correcta de respuesta a situaciones generalizadas 0 , en cualquier caso, estructurales y mantenidas en el tiempo, duraderas, crónicas (Aguilar, 2014: 26).

La respuesta a la exclusión social se organiza a partir del agrupamiento por categorías de estas situaciones ante las que reaccionar, y desde la lógica de que solo es exclusión aquello que se vincula a pobreza material vinculada al acceso a recursos y servicios, y ausencia de apoyo social desde las visiones, aún arrastradas, de la desviación social: son excluidos o excluidas quienes arrastran una etiqueta estigmatizante. Si no la llevan, son "otra cosa”, no son personas excluidas y, por tanto, son tributarias de otro tipo de apoyo social. A pesar del titánico esfuerzo generado desde dentro de los servicios sociales para cambiar esta aproximación conceptual, sigue siendo la predominante, entre otros motivos, porque sigue siéndolo para la mayoría de la sociedad y para el sentido que orienta las leyes y enfoque de las políticas públicas en materia social. Aguilar, de nuevo, dice sobre los servicios sociales: "se entendían no tanto como un pilar del Estado social, sino como un último nivel" (Aguilar, 2014: 19). Nos atrevemos a sugerir que efectivamente se ha avanzado en la comprensión a nivel teórico del sistema de protección social como uno de los pilares del Estado, pero siguen concretándose en gran manera desde un modelo asistencialista que los y las profesionales, así como las personas afectadas, luchan por subvertir.

Una de las consecuencias, que no la única, de esta manera de entender la exclusión social es la necesidad de situar en subsistemas distintos el acompañamiento y respuesta de los sistemas de servicios sociales: por un lado, la enfocada a la respuesta a las necesidades de la exclusión social; por otro, la respuesta a las necesidades sociales distintas a lo que suele categorizarse como exclusión social. Ese sería uno de los rasgos que históricamente ha diferenciado los servicios sociales básicos de los especializados, o los de primaria relacionados con los de secundaria, según la terminología que se utilice. No obstante, la complejidad de la que antes hablábamos está ayudando a comprender el error de base de este abordaje: cada vez más personas evidencian situaciones cruzadas que hibridan situaciones 
-y por tanto necesidades de respuesta- de uno y otro subsistema, llevándonos cada vez más a comprender que la especificidad creciente de cada persona solo puede ser abordada desde sistemas flexibles y capaces de abordar cada situación y su particularidad.

A partir de este análisis, resulta muy interesante la propuesta del País Vasco, que entiende que toda situación tributaria de recibir prestaciones del sistema de protección social, sin excepciones, se enmarca en un contexto de exclusión social. Y entiende que es así porque define "exclusión social" como aquellas condiciones de vida sostenidas en el tiempo que generan una situación de la ausencia suficiente de recursos personales, relacionales y materiales. No es casual que esta conceptualización del concepto de exclusión social se vincule a través del Decreto 385/2013 por el que se aprueba el Instrumento de Valoración de la Exclusión Social del País Vasco al concepto de interacción (Fantova, 2017: 14). Este concepto, que el autor define como "el ajuste entre la capacidad de desenvolvimiento autónomo de la persona en sus entornos vitales y el apoyo social disponible a través de los vínculos familiares, convivenciales, comunitarios y sociales en general” (Fantova, 2007: 9), como él mismo afirma, hace referencia a la persona y su situación, pero también al apoyo que recibe, a los vínculos de que dispone y a la autonomía posible de la persona en su circunstancia.

Esta mirada permite salir de la lógica clásica de la exclusión social y centrarse en un nuevo abordaje que es compatible con las necesidades de respuesta, cada vez más complejas. La intervención social sería, según Fantova: "la actividad que tiene como finalidad prevenir, corregir o paliar desajustes en lo relativo a la interacción de las personas, con sus dos caras o dimensiones: autonomía funcional e integración relación" (Fantova, 2017: 13).

Un buen ejemplo de por qué el concepto de interacción concreta la posibilidad de entender la exclusión social como transversal a toda respuesta y apoyo de acompañamiento social, sean servicios básicos o especializados, nos lo ofrece el autor cuando afirma que: "Según esta mirada, los servicios sociales no deben poner el foco en la manera en que la discapacidad de una persona afecta (o no) a su empleo, alojamiento o ingresos, sino a su autonomía en las actividades de la vida diaria, al autogobierno de su vida, a sus vínculos familiares o a sus relaciones comunitarias. Que son exactamente los mismos aspectos que interesan a los servicios sociales en el caso de una persona en situación de grave exclusión social” (Fantova, 2017: 16). En definitiva, el concepto de interacción conectaría con los planteamientos de Martha Nusbaumm y Amartya Senn relativos a centrase en la capacidad de la persona de hacer y de ser. Y lo haría desde un enfoque positivo: centrándose no tanto en dotar a la persona de aquello que debería tener a su alcance, como en recuperar o apoyarla a ser $\mathrm{y}$ hacer en base a quien es.

\section{Complejidad: un concepto aún por definir, una realidad aún por estructurar}

Una vez más el punto de partida, la definición del concepto, juega en contra. Si bien el recorrido del concepto de exclusión social ha sido largo, dinámico y no ha implicado aún una concreción consensuada de su aplicación a las situaciones reales que define, el concepto de complejidad en el ámbito social ni siquiera ha sido abordado a fondo, tampoco desde la investigación científica. La complejidad resulta evidente en la práctica de los servicios sociales, se acepta no solo su existencia sino también su trascendencia e importancia crítica para poder estructurar los sistemas de servicios sociales, pero no hemos estudiado ni objetivado qué es ni qué representa en contexto de necesidades sociales y la organización de la respuesta a estas. Se sobreentiende su significado y se articula de forma arbitraria en cada servicio social. No es así en el ámbito sanitario, que cuenta con abundante literatura científica, nacional e internacional desde inicios de los años 1980. Sus conclusiones no son transportables a los sistemas de servicios sociales puesto que su elevado nivel de desarrollo y concreción en cada sistema sanitario le da naturaleza de alta especificidad para cada sistema en el que se desarrolla, haciéndolo desde las lógicas, sistemas y herramientas de éste ámbito y enfocado básicamente -aunque no solamente- a la salud.

No obstante, y desde el reconocimiento de que el abordaje de la complejidad en situaciones de salud pasa también por un abordaje social, el ámbito sanitario ha llevado a cabo un gran desarrollo en las últimas dos décadas tanto de la respuesta a las necesidades sociosanitarias, como de la interacción del sistema de salud con el ámbito meramente social de las personas en situaciones de complejidad en la salud. Este encaje establece puntos de encuentro entre la visión de la complejidad del ámbito sanitario con la del ámbito social, aún pendiente de construcción formal a pesar de su presencia en el día a día. En este sentido, nos permitimos tomar algunas ideas de Maria Josep Lòpez y Sebastià Santaeugènia que deben ayudar a orientar el abordaje de la complejidad desde lo social (Lòpez y Santaeugènia, 2018). La persona como centro del sistema es la primera de ellas, ampliamente consensuada en los dos ámbitos, sanitario y social, pero aún pendiente de una real aplicación -al menos en el ámbito de los servicios sociales-. La doble orientación sanitaria y social, es, también, aceptada desde el consenso. El empoderamiento de la persona como parte de la construcción del acompañamiento - sanitario o social-del que va a ser objeto, es otro, pero no debe escapársenos la profundidad de este postulado: dar a la persona la titularidad de la toma de decisiones en su acompañamiento social, es, nos atrevemos a decir, revolucionario - y una revolución necesaria, por otra parte-, a tenor de lo que se recoge en la investigación en curso sobre el relato de práctica de los equipos profesionales que trabajan con personas en situación de sinhogar en un programa de atención 
que aplica el modelo Housing First (Llobet et al., 2018). Otra de las cuestiones que plantean es entender la comunidad en la que las personas viven como un dispositivo per sé, con agencia y que apoya la propia agencia de la persona. Por tanto, habría que facilitar en contexto comunitario elementos de proximidad -inserción o capacidad de respuestade los ámbitos sanitario y social en su seno, previa definición de cuáles son los núcleos comunitarios de abordaje - barrio, grupo de calles, pueblo, otras categorías-. También visibilizan las situaciones de complejidad como transversales a todo el espectro de la sociedad: una parte de la misma no tiene problemas de salud, pero la que los tiene, oscila entre situaciones de baja complejidad, de bastante complejidad o condiciones de avanzada complejidad: lo mismo sucedería en el ámbito de los servicios sociales, con un elevado porcentaje de las personas tributarias del sistema de protección social con necesidades complejas de una u otra intensidad, y oscilaciones entre ellas en función del momento personal de cada sujeto. Finalmente, cabe destacar que estos autores entienden que la interrelación entre sistemas poco adaptados a la necesidad de las personas favorecen las situaciones de complejidad - lo cual debería ser abordado urgentemente desde los sistemas de servicios sociales si no queremos contribuir a añadir complejidad y a cronificarla al no haber adaptado la interrelación entre sistemas a esta complejidad que reconocemos como estructural y crítica. Lòpez y Santaeugènia hablan -y disponentambién de herramientas, pero: ¿cómo disponer de herramientas desde el ámbito social cuando aún ni se ha formalizado qué es y cómo se materializa la complejidad en el ámbito social, estando aún trabajando el consenso en la concreción de herramientas para la exclusión social? En cualquier caso, está aún pendiente de acotación el marco - a concretar- de lo que podríamos definir como complejidad desde el sistema de protección social.

Tomemos como punto de partida que el objetivo de todo servicio social será siempre y en todo caso, acompañar las situaciones - o riesgo de situaciones- de exclusión social. Proponemos aceptar como significado primario de complejidad aquello que se compone de elementos diversos, 0 el conjunto o unión de dos o más cosas. A partir de estas dos premisas, proponemos un primer marco de la complejidad a partir de la experiencia, reflexión y propuesta llevada a cabo en el País Vasco. Así, pasamos aquí a entender como complejas -o como atención a la complejidad- en el ámbito social:

- Todas las situaciones de exclusión social pueden, per sé, conllevar complejidad media o alta, en función de la valoración de la diversidad de elementos que estructuran cada situación personal de exclusión social.

- Todas las situaciones de exclusión social (leve a grave), que se combinan con situaciones de desprotección -menores de edad o personas adultas en manifiesta incapacidad-, y/o con situaciones de dependencia entendida en un sentido amplio (dependencia, discapacidad física, psíquica, problemas de salud mental).

Por tanto, las categorías genéricas que se proponen en relación a la complejidad, son las siguientes:

- Exclusión social leve, moderada o grave, como complejidad media o alta.

- Exclusión social más dependencia, leve o moderada las dos o cualquiera de las dos, complejidad media.

- Exclusión social más dependencia grave, las dos o cualquiera de las dos, alta complejidad.

- Exclusión social más desprotección, alta complejidad.

- Exclusión social más desprotección más dependencia, alta complejidad.

\section{La atención a la exclusión social desde la interacción y respondiendo a la complejidad: la permeabilidad entre servicios sociales básicos, especializados y el papel de la atención comunitaria}

Como se ha dicho ya, la división entre servicios sociales básicos y especializados, entendida como una separación de abordajes entre necesidades sociales derivadas de la exclusión social -básicos-; y necesidades sociales no relacionadas con la exclusión social tanto cuanto relacionadas a la especialidad de la respuesta - especializados-, parece haber perdido el sentido que hubiera podido tener en el pasado. Fantova apunta la obsolescencia de este abordaje, más cuanto la personalización de la atención se opone directamente a esta dicotomía básico o primaria y especializado o secundaria, resultando "[...] inadecuados y disfuncionales como criterio de segmentación para unos servicios sociales universales que pretendan proteger y promover la interacción de todas las personas con una orientación personalizada, preventiva, comunitaria y poblacional en una sociedad del conocimiento, del riesgo, del bienestar y de la complejidad" (Fantova, 2017: 22).

La atención centrada en la persona no puede entenderse más que desde sistemas en conexión y desde circuitos e itinerarios personales reales y posibles, lo cual no existe. En cambio, la concreción de la exclusión social desde la lógica de la interacción aplana el camino para que esta conexión se dé. Y la respuesta no solo a la complejidad, sino también a su condición cambiante - por su capacidad de ser creciente o decreciente en cada caso, así como de poder cambiar en cada persona y momento lo que configura la complejidad-, lo hace imperativo. Deben construirse espacios de intersección entre lo que hoy asumen, con fronteras cerradas, los sistemas de primaria y secundaria. Pero eso no basta: no se trata en ningún caso de crear una tercera estructura "intermedia" entre una y otra, sino 
de entender que la complejidad es potencialmente transversal a toda situación de exclusión social. Que, por tanto, las diferencias de respuesta entre básico y especializado deben difuminarse, y los circuitos, protocolos y normativa de aplicación en base a cada caso, flexibilizarse. Abandonar la definición del funcionamiento de los sistemas desde la caracterización de los recursos, centros, dispositivos disponibles, para pasar a hacerlo a partir de los circuitos e itinerarios personales necesarios para dar respuesta a las situaciones individuales en orden a la recuperación y mantenimiento de la interacción.

El cambio es, por tanto, de modelo. E implica un cambio profundo, posiblemente, el mayor desde los años 1990. A este cambio, hay que añadir el ya inexcusable aplazamiento de la incorporación de la atención comunitaria, una de las grandes asignaturas pendientes de los sistemas de servicios sociales y con un peso de valor igual al de las respuestas articuladas desde la que debería ser reformulación de los sistemas básicos y especializados.

La personalización cualificada de la atención, junto a la proximidad comunitaria, son la única alternativa posible a "la baza de la intensidad (horas) de prestación profesional de menor valor añadido" (Fantova, 2017: 20). Una intervención comunitaria que debe ser entendida como un método complementario al servicio de las intervenciones con personas (Aguilar et al., sin fecha: 83). No es casual que el trabajo comunitario sea, por sí mismo, una de las finalidades con las que surge el actual sistema primario de los servicios sociales (Aguilar et al., 2012: 20), si tenemos en cuenta que la misión de los servicios sociales es apoyar la cohesión, integración y justicia sociales. El abordaje desde la atención comunitaria: al incrementar las conexiones sociales; desarrollar el apoyo mutuo entre personas; incorporar socialmente a las personas en situaciones de exclusión social; desarrollar la capacidad de interlocución y negociación de los agentes implicados apoderándoles, entre otros (Aguilar et al., 2012: 19), juega un imprescindible papel en relación a la interacción como objeto de la intervención social. Dicho de otra manera: sería muy difícil, cuando no imposible, abordar estos objetivos ni alcanzar la interacción deseada en cada caso de exclusión social acompañado desde los servicios sociales, sin una atención comunitaria incorporada a los servicios sociales de forma plena.

\section{Rellenando espacios: hacia una nueva organización de los sistemas de servicios sociales}

Como punto de partida de este apartado, diremos que a partir de la misión de contribuir a la justicia social, la integración y la cohesión social (Roman, 2016: 13), los servicios sociales tendrían como objetivo siempre y en todo caso revertir las situaciones de exclusión social. Que se entendería esta -la exclusión social-como la insuficiencia en la capacidad de desenvolvimiento autónomo -físico, comunitario, social-de las personas en sus entornos vitales, insuficiencia a partir de la cual estructurar la acción de los servicios sociales (Fantova, 2008), y considerando la complejidad de las situaciones como eje transversal y punto crítico que define hoy en día la capacidad y efectividad de la respuesta. Que esta respuesta debería organizarse a partir de la redefinición interna y de relación de los sistemas básicos y especializados, y la incorporación de la atención comunitaria como un dispositivo central del acompañamiento (Aguilar et al., s.f.: 2012; Aguilar: 2014). Y que esta organización debería darse a partir de conceptos e indicadores consensuables que permitan generar estructuras replicables en todos los servicios sociales, e interoperables con otros sistemas, como el sanitario y el educativo, entre otros.

Estamos proponiendo, pues, aceptar la exclusión social como único objetivo de los servicios sociales, pero comprendiendo que son situaciones de exclusión social no aquellas históricamente vinculadas a la estigmatización originada por los discursos del "fracaso" o la "desviación” sociales, sino aquellas situaciones que imposibilitan la capacidad de interacción de la persona. En ningún caso se propone sustituir exclusión social por interacción, sino entender la exclusión social a través de la interacción. Abandonar el marco lógico actual, orientado por el viejo modelo de la caridad y la desviación social, y dar respuesta a las situaciones de exclusión social a través del trabajo sobre situaciones de vulnerabilidad que afecten a la capacidad de autonomía personal en el contexto social. Incidiendo así en la cohesión social desde el apoyo a individuos en situaciones vulnerables, en lugar de incidir en los individuos para que se adecuen o acomoden al statu quo social normativo.

Estamos, también, destacando el rol prioritario y crítico de una estructuración sistematizada en la que se tome en consideración la atención a las situaciones complejas como elemento clave del abordaje: entender la exclusión social a través de la interacción, materializando la actuación desde la complejidad (su ausencia o su presencia y sus niveles y ritmos). Sirvan como ejemplo los resultados de un interesante estudio realizado en el País Vasco por Izaskun Ormaetxea. A partir del ya citado Decreto 385/2013, que aprobó el Instrumento de Valoración de la Exclusión Social (IVES) y a partir de su categorización de los conceptos de exclusión social, desprotección y dependencia, toma una muestra de 181 personas y detecta que la categoría exclusión social como única, afectaba a un 20,4\% de la muestra; la combinación de exclusión social más desprotección a un 2,8\%; la combinación de dependencia más exclusión social a un 59,1\%; y la de exclusión social más desprotección más dependencia, a un 17,7\% (Ormaetxea, 2018).

Estos datos, de carácter indiciario - quizá no probatorio-, en cualquier caso de gran interés, nos orientan sobre qué queremos decir cuando 
hablamos de complejidad y nos dan pistas sobre el peso de la complejidad en el contexto global del acompañamiento al que se deben los servicios sociales: una quinta parte de la muestra, el 20,4\%, se corresponde a una única categoría, la de exclusión social. Así pues, la gran mayoría, casi un $80 \%$ del total, son por definición situaciones de complejidad fruto de la combinación de dos o más de las categorías. Este abordaje nos remite a una visión más horizontal que vertical. Es decir, más a unas situaciones en paralelo que pueden sobreponerse y hacerlo, además, de manera rápida en cualquier sentido -apareciendo o despareciendo-, que a situaciones jerarquizadas en las que una situación tenga que gobernar o decidir sobre las otras, de manera básicamente permanente. Como siempre, la realidad social es tan diversa que todas las posibilidades caben. Por supuesto, hay situaciones que piden una respuesta jerarquizada y que tendrán poca o nula rotación orientada al cambio del abordaje a realizar. Pero todo parece apuntar que las estructuras flexibles y horizontales son las que tendrán que dar más volumen de respuesta y serán las más bien posicionadas para gestionar de manera útil y satisfactoria recursos, circuitos y dispositivos que potencien la interacción, y hacerlo tomando en consideración la capacidad de agencia de los individuos y de la comunidad.

\section{Primaria, secundaria... básicos, especializados... ¿superamos ya la dicotomía?}

Fantova opina que la diferencia entre uno y otro subnivel debería estar en la intensidad de apoyo, y que su denominador común estaría en que ambos abordan discapacidad y/o dependencia, desprotección y exclusión (Fantova, 2017: 15). Aguilar afirma que los servicios sociales - la totalidad de ellos, independientemente de sus subsistemasdeberían focalizarse en tres campos: dependencia y cuidados de larga duración; menores y sus familias; procesos de incorporación y (re)inserción social (Aguilar, 2014: 32). Este autor afirma también que la atención domiciliaria es "probablemente la [en cursiva en el texto original] pieza clave, de un sistema sólido de cuidados de larga duración” (Aguilar, 2014: 27), lo cual orienta a una perspectiva que difiere de la atención de larga duración en dispositivos rígidos, para centrarse en un abordaje en otro tipo de dispositivos o sin ellos, y siempre desde lo comunitario, favoreciendo así el trabajo enfocado a posibilitar y potenciar la capacidad de interacción.

Ambos autores, de manera confluyente y complementaria, parecen orientar su propuesta hacia servicios sociales organizados en subsistemas paralelos y más diferenciados por la intensidad del acompañamiento social que por la categorización de la situación de la persona. Esta visión transversaliza y acerca mucho el objeto de dedicación de dos subsistemas históricamente tan separados como el básico y el especializado. A su vez, la incorporación del papel clave de la atención domiciliaria y sus posibilidades de mantenimiento o restablecimiento de la autonomía y agencia de la persona, también deberían trabajarse de manera indistinta tanto desde lo básico como desde lo especializado, al entenderse que es su intensidad y no su tematización lo que definiría uno u otro acompañamiento. Esto nos lleva a un gran espacio de confluencia entre lo que hoy se entiende como básico o especializado, primaria o secundaria. Y también, y, sobre todo, a un cambio absoluto en relación a la transversalización de ambos.

\section{Una propuesta orientada al cambio}

¿Cómo podemos aterrizar estas propuestas, en forma de modelo que pudiera ser sistematizado, replicable e interoperable con sistemas distintos? Proponemos, a partir del análisis y las sugerencias de Manzano (2018, s.f.), tomar como punto de partida la pirámide de Kaiser -o pirámide de estratificación permanente del riesgo de Kaiser-. Sobre todo, porque hacerlo favorece la posibilidad de establecer una buena base de interoperatibilidad entre los sistemas social y sanitario. En cualquier caso, a partir de la estructura básica de la pirámide de Kaiser, lo que aquí se propone pretende ser una aproximación al debate sobre cómo concretar la organización interna de los sistemas los servicios sociales. La pirámide escala de forma progresiva considerando, de menos a más, las situaciones de no complejidad o baja complejidad; complejidad media; alta complejidad.

\subsection{No complejidad o baja compejidad}

Tomando la experiencia del País Vasco, las situaciones de no complejidad o baja complejidad, relativas al riesgo de exclusión social, se abordarían tomando los criterios 1 y 2 -respectivamente, carencias relacionales y personales clave; merma de la autonomía vital-, así como las situaciones de dependencia sin riesgo de exclusión social. ¿Cómo? Acompañando la autogestión de la situación por parte de cada persona con actuaciones intensivas de los servicios sociales básicos y/o actuaciones intensivas y planificadas a nivel comunitario, y posibilidad de incorporar atención domiciliaria, seguramente de baja intensidad y en poco volumen de casos.

\subsection{Complejidad media}

Las situaciones de complejidad media implicarían las de exclusión social leve o moderada, así como las de exclusión social leve o moderada, combinadas con dependencia leve o moderada. ¿Cómo? También aquí la autogestión tiene cabida, acompañada de una atención comunitaria y/o de una gestión de caso - con todo lo que implica el concepto de gestión de caso a nivel de referente único y atención centrada 
en la persona- que debería poder ser asumida tanto desde la básica o desde la especializada, en función de las necesidades de cada situación y momento. La atención domiciliaria puede ser uno de los pilares fundamentales de la complejidad media.

\subsection{Alta complejidad}

La alta complejidad comprendería la exclusión social grave; las situaciones de exclusión social y dependencia en que una de las dos o las dos, sean graves; y todas las situaciones en las que se combine desprotección y exclusión social -es decir, con o sin dependencia-, al implicar las situaciones de desprotección de por sí alta complejidad al ser normalmente relativas a menores de edad o a personas con la capacidad de decisión severamente limitada. ¿Cómo? Mayoritariamente la vía será la de la gestión de caso, pero sin descartar la posibilidad de una articulación comunitaria, ya sea en forma de simbiosis entre equipamientos y dispositivos sociales de alta especialización con una potente imbricación y vinculación con el trabajo comunitario, ya sea en forma de atención domiciliaria altamente especializada también vinculada a la atención comunitaria.

\subsection{Carteras de servicios, circuitos, reglamentación}

Se ha venido insistiendo en que en ningún caso se trataba de fundir los dos subsistemas en uno, sino de definir un nuevo modelo que, en la totalidad de sus subniveles, permita un tránsito ágil y una adecuación a la necesidad de cada persona. Por eso los canales de comunicación y trasvase de uno a otro subsistema en base a las particularidades y cambios en la situación de complejidad de las personas son tan importantes como su estructuración en sí misma. Eso se traduce en circuitos de transición muy ágiles que den respuesta efectiva a la necesidad de que las personas transiten de una a otra respuesta a su situación de complejidad: no deberá ser posible no pasar de forma directa y rápida de una situación de inclusión a una de no complejidad o baja complejidad, o a una de complejidad media, o a una de alta complejidad. No deberá ser posible no pasar de la de no complejidad o complejidad baja a la de alta complejidad directamente. Y, por supuesto -y sobre todo- no deberá ser posible el pasar de nuevo a otra de las situaciones, ya transitada. Si bien esto pudiera parecer pura abstracción sobre el papel, sin afectación sobre las personas y sus necesidades, es en cambio la piedra de toque de la capacidad (o incapacidad) del acompañamiento de la

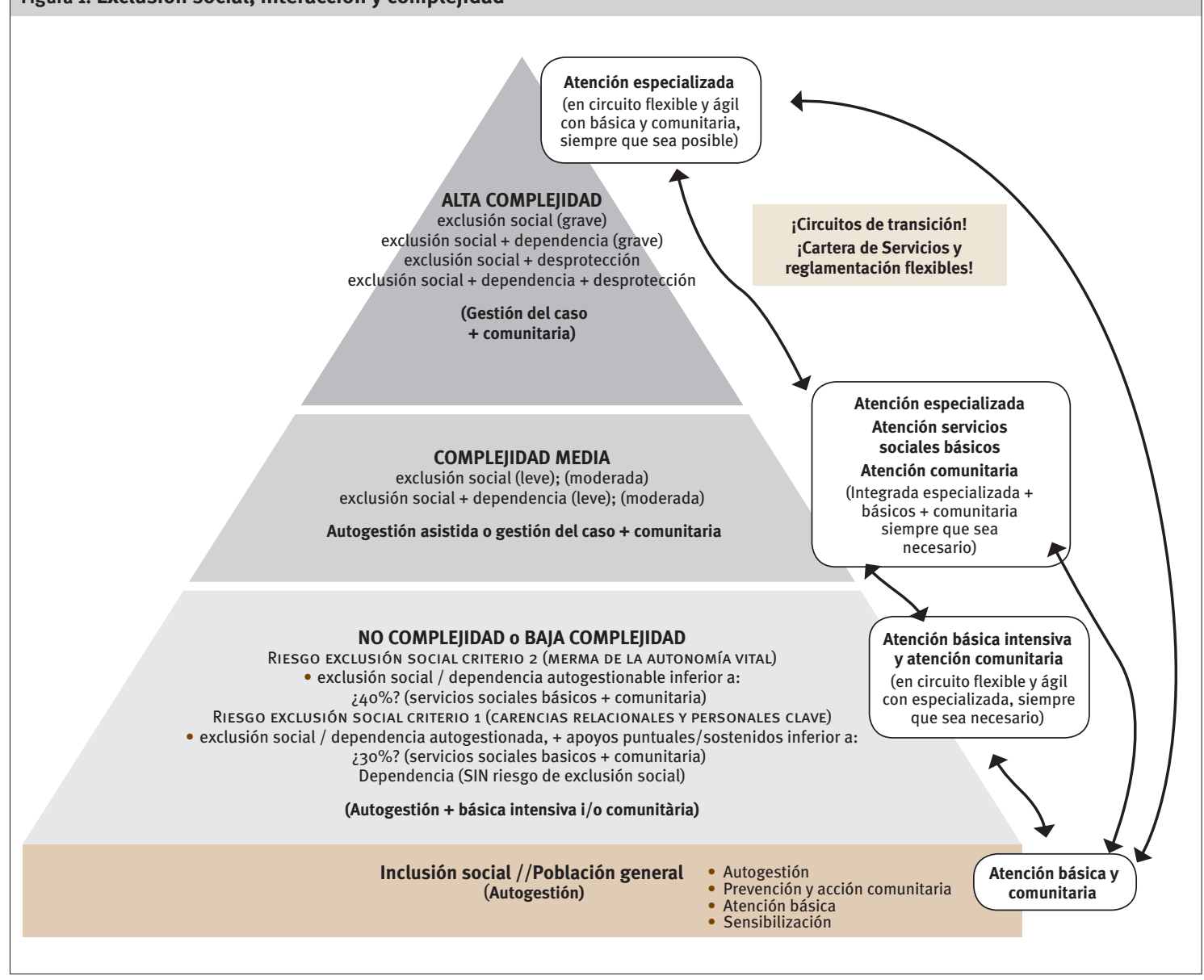

Fuente: Elaboración propia. 
complejidad en las situaciones de exclusión social: si una persona en situación de alta complejidad lleva a cabo una mejoría que la sitúa en una de no complejidad o baja complejidad, o de complejidad media, no se le puede no dar respuesta a esta adecuación o tardar años en hacerlo. Todo ello debido a que los circuitos no permitan moverse de uno a otro tipo de acompañamiento a riesgo de perder todo derecho de asistencia social en el caso de que sobrevenga otro cambio en la situación personal, debido a la incapacidad del sistema de absorción de la movilidad, y de relajar los criterios de la reglamentación y definición de circuitos, basados en la gestión de los escasos recursos disponibles y no en la necesidad cambiante de las personas. El funcionamiento actual del sistema de servicios sociales está jalonado de estas trabas mayúsculas que deben ser superadas con carácter de urgencia.

Esta "revolución" - la de la flexibilización normativa de reglamentos y circuitos-, es de tanto calado en magnitud y trascendencia como la de la adaptación de la cartera de servicios, adaptándola a los criterios del acompañamiento desde recursos flexibles, de inserción comunitaria y, en lo posible, de atención domiciliaria.

\section{Recursos a explorar, temas a abordar}

La atención centrada en la persona y la orientación del abordaje de la exclusión social a través de la interacción, implica por definición un cambio de las carteras de servicios, potenciando los equipamientos de pocas plazas residenciales y de marcada orientación a un funcionamiento potenciador de la autonomía, los recursos y servicios vinculados al domicilio de la persona: enfoques orientados a potenciar la agencia de las personas y su integración en dinámicas de acompañamiento comunitario. Los circuitos y la reglamentación relativa al funcionamiento y requisitos de los servicios, recursos y prestaciones sociales, deben reformularse a partir de la ya urgente necesidad de acompañar los cambios de situación de las personas, en lugar de enfocarse en gran medida, y por encima de las personas, en el funcionamiento y la gestión de los dispositivos y recursos que se les proporciona a estas. Redimensionar los servicios sociales desde lo aquí propuesto tiene en los consorcios un contexto natural en el que implementar y asentar este encaje que va más allá de superar la dicotomía entre las dos visiones del acompañamiento social, el básico y el especializado: también les permite actuar como encaje de marcos legales y normativos distintos -los municipales y los autonómicos-; marcos y objetivos distintos - prestar servicios a vecinos de un municipio, o hacerlo a los ciudadanos de una comunidad autónoma-.

Las y los profesionales, piezas clave de la orientación del cambio, serán también objeto del cambio a sistematizar en su práctica profesional, definida a partir de modelos y sistemas en los que se arrastra hasta el momento la tensión entre la persona a acompañar, que no acaba de ser posible poner en el centro del proceso y asumiendo el liderazgo del mismo, sino que lo era el sistema de servicios sociales. Por ello, serán punta de lanza del cambio, pero también primera línea a la que acompañar intensamente en su adecuación al cambio, potenciando sus capacidades y minimizando el impacto sobre las personas que conforman los equipo de profesionales. Sigue pendiente de abordaje la aún inexistente definición consensuada de los conceptos tratados -exclusión social, complejidad, dependencia en un sentido amplio, desprotección, y los límites de las graduaciones de todos ellos en tanto que afectación leve o baja, media, alta-; sus indicadores, definidos, medibles y también consensuados, $y$, a su vez, compatibles con los de los sistemas sanitario y educativo. Y, a partir de estas definiciones y parametrizaciones, la elaboración de herramientas comunes, compatibles a nivel de interoperatibilidad y transportables a sistemas de información integrados.

No podemos desfallecer. Los derechos sociales, derechos humanos de segunda generación, se materializan solo si se implementan los recursos y servicios sociales que cada sociedad necesita en un momento determinado. En nuestras sociedades, siempre sujetas a un cambio cada vez más rápido, no hay más opción que asumir la siempre urgente necesidad de cambio, y ejecutarla. Sin más demora. ¡Manos a la obra! 


\section{Bibliografía referenciada}

AGUILAR, M. (2014): "Apuntes para un replanteamiento de los servicios sociales en España”, VII Informe sobre exclusión y desarrollo social en España 2014, Fundación FOESSA.

AGUILAR, M.; CORERA, C.; LAPARRA, M.; LIBERAL, B. y PÉREZ, B. (sin fecha): “Capítulo 2. Una respuesta de hoy para el futuro. La reordenación de la atención comunitaria de servicios sociales", Documento base para un plan de atención comunitaria de servicios sociales, Gobierno de Navarra.

AGUILAR, M.; LLOBET, M. y PÉREZ, B. (2012): “Los servicios sociales frente a la exclusión”, Zerbitzuan, nํ51, pp. 9-26.

AYUNTAMIENTO DE BARCELONA (2017): Estrategia de Inclusión y de Reducción de las Desigualdades Sociales de Barcelona (2017-2027) http:// www.bcn.cat/barcelonainclusiva/ca/2018/6/ estrategia_inclusion_2017-2027_es.pdf [consultado el 22 de febrero de 2019].

BRUGUÉ, J.; GOMÀ, R. y SUBIRATS, J. (2002): “De la pobreza a la exclusión social”, Revista Internacional de Sociología, nํㅜ 33, 2002, pp. 7-45.

CASADO, D. (2014): “Los servicios sociales públicos”, VII Informe sobre exclusión y desarrollo social en España 2014, Fundación FOESSA.

CASTEL, R. (1997): Las metamorfosis de la Cuestión Social. Una crónica del salariado, Paidós, Buenos Aires.

DEPARTAMENT D'ACCIÓ SOCIAL I CIUTADANIA; GENERALITAT DE CATALUNYA (2010): Diccionari de Serveis Socials, Termcat http://treballiaferssocials. gencat.cat/web/.content/o1departament/ o8publicacions/ambits_tematics/serveis_ socials_2011/o1diccionari/diccionari.pdf [consultado el 22 de febrero de 2019].
DEPARTAMENT DE TREBALL, AFERS SOCIALS I FAMÍLIES, GENERALITAT DE CATALUNYA (sin fecha): http:// treballiaferssocials.gencat.cat/ca/ambits_ tematics/pobresa_i_inclusio_social/ [última consulta: 22 de febrero de 2019].

EIZAGUIRRE, A.O. (2016): "Regulación del diagnóstico social en el País Vasco: intenciones, resultados y vericuetos", Zerbitzuan, no 61, pp. 21-32.

FANTOVA, F. (2007): "Repensando la intervención social”, Documentación Social, noำ 147, pp. 183-198.

- (2008): "Sistemas públicos de servicios sociales. Nuevos derechos, nuevas respuestas", Cuadernos Deusto de Derechos Humanos, nำ 49, Universidad de Deusto, Bilbao.

- (2017): "Servicios sociales e inclusión social: análisis y perspectivas en el País Vasco", Zerbitzuan, no 64, pp. 9-31.

GARCÍA, J. (Coord.) (2013): Exclusiones. Discursos, políticas, profesiones, Editorial UOC, Barcelona.

GENERALITAT DE CATALUNYA (2006): Decreto 318/2006, de 25 de julio, de los servicios de acogida residencial para personas con discapacidad, DOGC, 4685, de 27 de julio de 2006.

- (2007): Ley 12/2007, d'11 d'octubre, de Serveis Socials, DOGC, núm. 4990, 18 de octubre de 2007.

- (2010): Ley 14/2010, del 27 de maig, dels drets i les oportunitats en la infància i l'adolescència.

- (2010): Ley 25/2010, del 29 de juliol, del llibre segon del Codi civil de Catalunya, relatiu a la persona i la família.

- (2017): Decreto 150/2017, de 17 de octubre, de la atención educativa al alumnado en el marco de un sistema educativo inclusivo, DOGC, 7477, 19 de octubre de 2017. 
GOBIERNO DE ESPAÑA (1996): Ley Orgánica 1/1996, de 15 de enero, de Protección Jurídica del Menor, de modificación parcial del Código Civil y de la Ley de Enjuiciamiento Civil.

- (2006): Ley 39/2006, de 14 de diciembre, de promoción de la autonomía personal y atención a las personas en situación de dependencia.

- (2011): Real Decreto 174/2011, de 11 de febrero, por el que se aprueba el baremo de valoración de la situación de dependencia establecido por la Ley 39/2006, de 14 de diciembre, de Promoción de la Autonomía Personal y Atención a las personas en situación de dependencia.

GOBIERNO VASCO (2013): Decreto 353/2013, de 28 de mayo, de Ficha Social del Sistema Vasco de Servicios Sociales y del instrumento de diagnóstico social del Sistema Vasco de Servicios Sociales, BOPV, núm. 121, 26 de junio de 2013.

- (2013): Decreto 385/2013, de 16 de julio, por el que se aprueba el Instrumento de Valoración de la Exclusión Social, BOPV, núm. 149, 7 de agosto de 2013.

JAUMANDREU, G. (2005): “Mapa de coneixement sobre l'exclusió social”, Revista Barcelona societat, 13, «http://ajuntament.barcelona.cat/ dretssocials/sites/default/files/revista/ revista13-art2-8-mapa-de-coneixementexclusio-social.pdf> [consultado el 22 de febrero de 2019].

LAPARRA, M. y PÉREZ, B. (coords.) (2008), “Exclusión social en España. Un espacio diverso y disperso en intensa transformación", Madrid, Fundación FOESSA.

LAPARRA, M.; OBRADORS, A.; PÉREZ, B.; PÉREZ, M.; RENES, V.; SARASA, S.; SUBIRATS, J. y TRUJILLO, M.: (2007): "Una propuesta de consenso sobre el concepto de exclusión. Implicaciones metodológicas", Revista Española del Tercer Sector, $\mathrm{n}^{-}{ }_{5}$, pp. 15-57.

LARRIÓN, B.; LETURIA, F.J.; ZALAKAIN, J.; ZABALETA, N. (2018). "Elkar-EKIN: (re)construyendo en común la red de servicios sociales para la inclusión en Gipuzkoa”, Zerbitzuan, n- 67, diciembre 2018, pp. 51-65.

LLOBET, M.; AGUILAR, M. (2016): "El Housing first. El dret a l'habitatge dels més vulnerables", Barcelona societat: revista d'informació i estudis socials, nํ․ 20, pp. 16-34.

LLOBET, M.; URIBE, J.; BATLE, S.; SANCHO, J.; CARTOIXA, J. y GUIJARRO, L. (2018): “Relato de práctica: la conciencia discursiva sobre una acción social en transformación", III Congreso Internacional de Trabajo Social: El Trabajo Social en un mundo en transformación: ¿distintas realidades o nuevos relatos para la intervención?, Universidad de Deusto, Bilbao.

LÒPEZ, M.J.; SANTAEUGÈNIA, S. (2018): Presentación en Jornada: "Una mirada cap a les persones amb condicions cròniques a la ciutat de Barcelona. Ho fem tot? Ho tenim tot?", en Caixa Forum de Barcelona, 15 de Noviembre de 2018 http:// salutintegralbcn.gencat.cat/web/.content/50 jornades/2018/fragilitat/01-20181115-Modelcronicitat-futur-ENAPISC-PPAC.pdf [consultado el 22 de febrero de 2019].

MANZANO, M.A. (2018): “Kaiser, o l'ombra fosca dels serveis socials" (2018) http://lleiengel. cat/kaiser-o-ombra-fosca-serveis-socials/ [consultado el 22 de febrero de 2019].

MANZANO, M.A.; PAGÈS, É. y SOLÉ, M. (sin fecha): Estratificación y determinación de necesidades sociales. Experiencia aplicada en la Provincia de Barcelona (poster), Diputació de Barcelona, https://www. diba.cat/documents/14465/73155505/ P\%c3\%b2ster+estratificaci\%c3\%b3\%20 i+determinaci\%c3\%b3\%2onecessitats+socials. ICIC16.pdf/41bo125b-b3f8-4f4b-8ddc5a1e241af7e1 [consultado el 22 de febrero de 2019].

MARTÍNEZ VEIGA, U. (2008): "Genealogía del concepto de exclusión social, la situación europea”, Trabajo Social Hoy, primer semestre 2008, pp. 7-44.

ORBEGOZO, A. (2016): "Regulación del diagnóstico social en el País Vasco: intenciones, resultados y vericuetos", Zerbitzuan, no 61, pp. 21 -32).

ORMAETXEA, I. (2018): “Interacciones entre situaciones de necesidad: un análisis de los diagnósticos especializados de exclusión de Bizkaia e implicaciones para el diseño de la red", Cursos de Verano, Universidad del País Vasco, San Sebastian.

ROMÁN, B. (2016): Ética de los servicios sociales, Herder, Barcelona.

SELF SUFFICIENCY MATRIX (SSM): http://www. selfsufficiencymatrix.org/zrm-int.aspx [consultado el 22 de febrero de 2019].

SIIS Centro de Documentación y Estudios (2015): "Reordenación de la red de recursos y programas para la inclusión social de Guipuzkoa", Zerbitzuan, 59, pp. 5-38. 